\title{
Estimation and resolution of heat capacities: lattice heat capacity morphology मै
}

\author{
David A. Johnson ${ }^{\text {a }}$, Edgar F. Westrum, Jr. ${ }^{\mathrm{b}, *}$ \\ ${ }^{a}$ Department of Chemistry, Spring Arbor College, Spring Arbor, MI 49283, USA \\ ${ }^{\mathrm{b}}$ Department of Chemistry, University of Michigan, Ann Arbor, MI 48109-1055, USA
}

\begin{abstract}
To utilize heat capacity determinations in the chemical thermodynamic region effectively, it is essential to be able to resolve the "excess" contributions, be they electronic (e.g. Schottky), magnetic, order-disorder, or one of the named transitions (Anderson, Verwey, etc.), from the often predominant lattice heat capacity. This paper addresses recent developments in bridging the experimental/utilization gap and compares the recent high precision approaches with each other and with experimental work on homologous series and selected substances.
\end{abstract}

Keywords: Excess heat capacity; Heat capacity; Lattice heat capacity; Precision; Thermodynamics; Volumetric priority

\section{Introduction}

The Latimer approach has been helpful in estimating and correlating entropies for many compounds but was inadequate when attempts to estimate and correlate the entropies of isostructural compounds of the lanthanide series were made for resolving excess (Schottky) and lattice contributions. Grimvall has recently pro-

\footnotetext{
"Presented at the Czechoslovak-French-Polish Conference on Calorimetry and Experimental Thermodynamics: Applications to Contemporary Problems, Prague, Czech Republic, 4-7 September 1993.

${ }^{*}$ Corresponding author.
} 
vided a theoretical justification of the Latimer approach. An empirical volumetric priority scheme of Westrum and co-workers proves more successful here as the lanthanide contraction in volume is of opposite sense to the cationic mass increase with atomic masses, but elsewhere in the periodic system there is a loss correlation between ionic masses and ionic radii. Moreover, utilization of the Volumetric Priority on homologous series of isostructural, lanthanide compounds has enabled determination of magnitudes of Schottky contribution frequencies of accuracy comparable to those obtained spectroscopically.

Perhaps the best and most precise is the frequency dispersion approach of Komada and Westrum. This modification of the Debye one-parameter $\theta_{\mathrm{KW}}$ (characteristic temperature) approach and adding approximations to simplify the Bornvon Kármán details yields an essentially single parameter approach to accurately model the lattice contribution but additional parameters can be introduced. In principle, this (theoretical) approach models the frequency distribution in a Debyelike form but with the inclusion of dispersion (i.e. frequency dependence of the wave velocity). Computer (PC) solution of the $C_{p}$ defining equations (about 40 integrals with variable limits) quickly yields characterization temperature from the heat capacity morphology. After some experience in choosing parameters, lattice heat capacities can be deduced with high precision compared to other methods enumerated. The high precision fit of this model succeeds when the Debye model (as usual) fails. The validity of the Volumetric Priority is demonstrated for the isostructural $\mathrm{Ln}(\mathrm{OH})_{3}$ series and gives essentially identical results with the Komada-Westrum approach for the $\mathrm{Ln}_{2} \mathrm{~S}_{3}$ series.

\section{Estimation of entropies}

In order to estimate standard entropies $S$ at $298.15 \mathrm{~K}$ for solids (an important thermophysical quantity) as well as to interpret heat-capacity determinations in the "chemical thermodynamic region" (about 5 to $350 \mathrm{~K}$ ) effectively, it is essential to be able to resolve the "excess" contributions, be they electronic (e.g. Schottky), magnetic, order-disorder, or one of the named transitions (Anderson, Verwey, etc.), from the often predominant lattice heat capacity. This paper addresses recent developments in bridging the experimental/estimation/utilization gaps and compares the recent high precision approaches with each other and with experimental work on homologous series and selected substances.

\section{Latimer's methods}

The estimation aspect, which may be hoped to provide results over the range 1 to $10 \%$, has the longer history and will be treated first. For inorganic materials Latimer's method developed first in 1921 and subsequently in 1951 [1] is clearly the most popular even today. Latimer recommended representing the entropy of a compound at $298.15 \mathrm{~K}$ by $S=\Sigma\left(1.5 R \ln M_{i}-0.94\right)$, where $M_{i}$ is the atomic weight 
of the $i$ th atom of the compound. Subsequently (over several decades) typical anion contributions were incorporated with values dependent on the oxidation state of the cation. This approach has been helpful in estimating and correlating entropies for many compounds. Indeed, in a recent paper, Grimvall [2] has developed the mathematical aspects of Latimer's method showing that Latimer's functional dependence correctly expresses the explicit mass dependence of the entropy at high temperatures. Khodakovski [3] has outlined statistical procedures for optimizing the anionic contribution and has argued in favor of a volume normalization prior to a cationic mass fitting. Cantor [4] likewise argued for a greater concern for volume.

\section{Other approaches}

Other schemes do exist, e.g. the closely related one of Wenner [5] and indeed, others [6]. Noteworthy among them is that of Zhang [7] which for organic substances is far more effective than those of Benson [8].

However, the Latimer approach is deficient. In Fig. 1, based on critical evaluation on transition element chalcogenides [9], many entropies deviate from the mid-mass range in characteristic fashion. Most $3 \mathrm{~d}, 4 \mathrm{~d}$, and $5 \mathrm{~d}$ chalcogenide

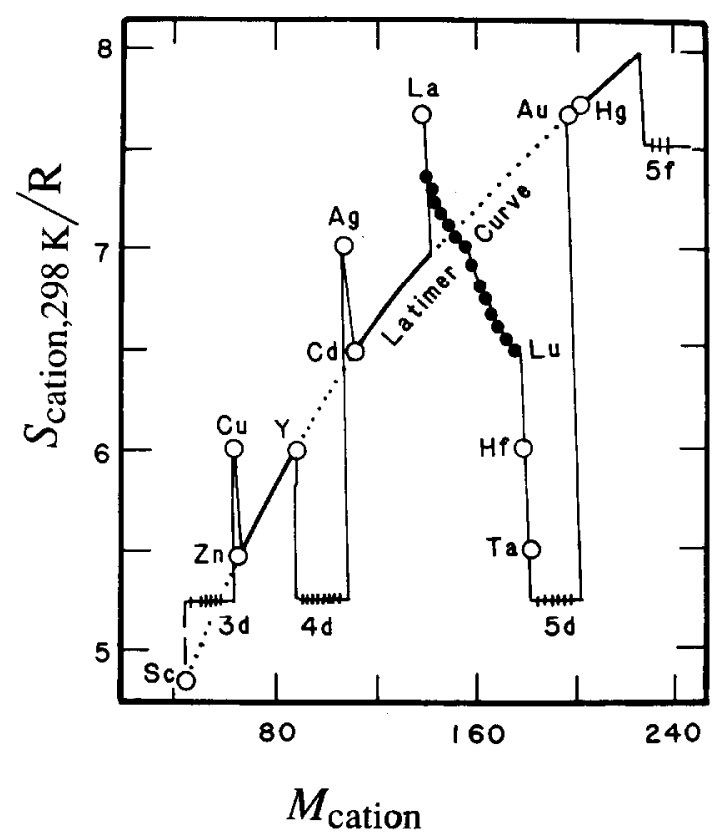

Fig. 1. A plot through the mid-portion of the periodic tables of the latticed contribution of a series of chalcogenides (including the lanthanides and the families of transition metal compounds). Based on data in Ref. [9]. 


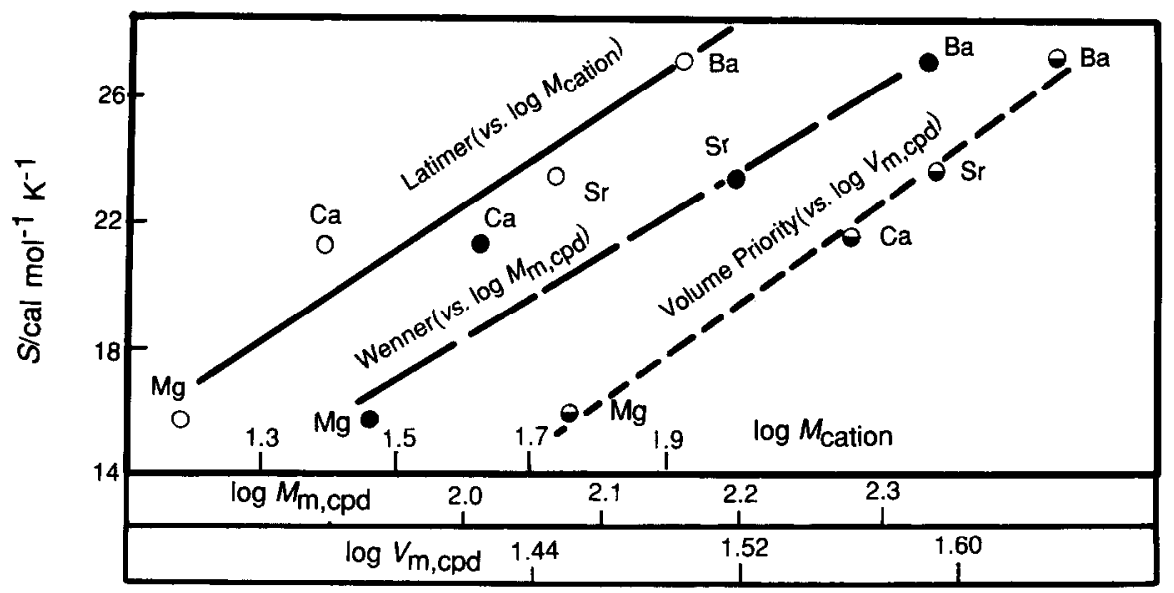

Fig. 2. Measured entropies of alkaline earth carbonates tested against several estimation theories $(1 \mathrm{cal}=4.187 \mathrm{~J})$.

compounds deviate most peculiarly from the logarithmic $M_{\text {cation }}$ curve and the $4 \mathrm{f}^{(1)}$ lanthanide compounds are perpendicular to the Latimer trend as a consequence of the well-known "lanthanide contraction." Through this region of the periodic table volume takes priority over mass, and the very empirical Volumetric Priority scheme (to be discussed subsequently) relates entropy (or at each temperature the heat capacity) as linear to $V_{\mathrm{m}}$. In Fig. 2 we compare the alkaline earth carbonate measured entropies against the three schemes and although the volumetric scheme is used out of context, it works better here than the others, but other sets show equally poor fits for all three schemes.

We have examined many aspects of this problem over the past months, the extent to which the cationic molar volumes $\left(V_{\mathrm{m}}\right)$ parallel the $M_{\mathrm{m}}$ values and the reliability of various schemes based on current data. For minerals and rocks the analytical approach of Kieffer [10] has proven quite productive.

\section{Resolution of lattice and excess heat capacities}

\subsection{Volumetric priority approach}

In the problem of the resolution of measured heat capacities, we first developed the Volumetric Priority scheme for iso-structural crystals already referred to in order to evaluate the so-called lattice heat capacities and on this basis to resolve the excess heat capacities. Briefly, the method involves a ratio of linear interpolations at each temperature of heat capacity (or other thermal functions) $C$, the molar volume $V$, for homologous isostructural compounds

$$
x=\left(V_{\alpha}-V_{\mathrm{d}^{\prime}}\right) /\left(V_{\mathrm{d}^{\prime \prime}}-V_{\mathrm{d}^{\prime}}\right)
$$


and

$$
C_{\alpha}=x\left(C_{\mathrm{d}^{\prime \prime}}-C_{\mathrm{d}^{\prime}}\right)
$$

using the volumes of the compound of interest and a diamagnetic compound $\mathrm{d}^{\prime}$; and that of the volume of the diamagnetic "end-point" compounds $\mathrm{d}^{\prime \prime}$ and $\mathrm{d}$ ' to define a coefficient $x$ which is then applied to $C$. This scheme which makes a linear interpolation between diamagnetic members of lanthanide series was tested initially by dilute doped samples, and as better spectroscopic samples became available by comparison of Volumetric Priority resolved Schottky contributions by thermophysical calorimetry with the spectroscopically ascertained low-lying crystalline electric field energy levels to about the accuracy between independent spectroscopic measurements [10]. Thus tested, the subsequent heat-capacity measurements were used to resolve $\mathrm{Ln}_{2} \mathrm{O}_{3}$ [12], $\mathrm{Ln}(\mathrm{OH})_{3}$ [11], $\mathrm{LnX}_{3}$ [13] ( $\mathrm{Ln}$ is lanthanide, $\mathrm{X}$ is halide) and $\mathrm{Ln}_{2} \mathrm{~S}_{3}[14,15]$ series Schottky contributions with excellent success. (However, only for the $\operatorname{Ln}(\mathrm{OH})_{3}$ homologous series does a single isostructural crystalline structure extend throughout the $4 \mathrm{f}$ elements.)

\subsection{Komada-Westrum approach}

Perhaps the best and most precise is the frequency dispersion approach of Komada and Westrum [16,17]. This modification of the Debye one-parameter $\theta_{\mathrm{KW}}$ (characteristic temperature) approach, with addition of approximations to simplify the Born-von Kármán frequency details, yields an essentially single parameter approach to accurately model the lattice contribution. Additional parameters can be introduced, or eliminated to default values.

In principle, this (theoretical) approach models the frequency distribution in a Debye-like form but with the inclusion of dispersion (i.e. frequency dependence of the wave velocity). Solution of the $C_{p}$ defining equations (about 40 integrals with variable limits) quickly yields a $\theta_{\mathrm{KW}}$ characteristic temperature from the heat-capacity morphology even on a fast personal computer within 10-15 s. After some experience in choosing parameters, lattice heat capacities can be deduced with high precision compared to other methods enumerated. The high precision fit of this model succeeds when modeling by Debye theory when the Debye approach fails. Comparison with experimental values for substances with only lattice heat capacity shows that $\theta_{\mathrm{KW}}$ is virtually constant over the range from 5 to $300 \mathrm{~K}$ (see Fig. 3). (At higher temperatures, an anharmonicity correction is needed.)

It should be noted in passing that Debye's neglect of the dispersion (i.e., variation of wave velocity with frequency) was in fact a serious over-simplification. The Barber and Martin [18] and the Barber and Westrum [19] modeling of glasses, the Kittel modeling of geomaterials, and the abortcd approach of Schrödinger [20] and the Komada-Westrum approach [16,17] are all a consequence of the recognition of this subtle, but very relevant, wave property.

In a recent paper [21] the volumetric priority method [11] for the isostructural $\mathrm{Ln}(\mathrm{OH})_{3}$ series has been demonstrated to give essentially identical results for the excess Schottky heat capacity as does the Komada-Westrum approach [16,17]. 


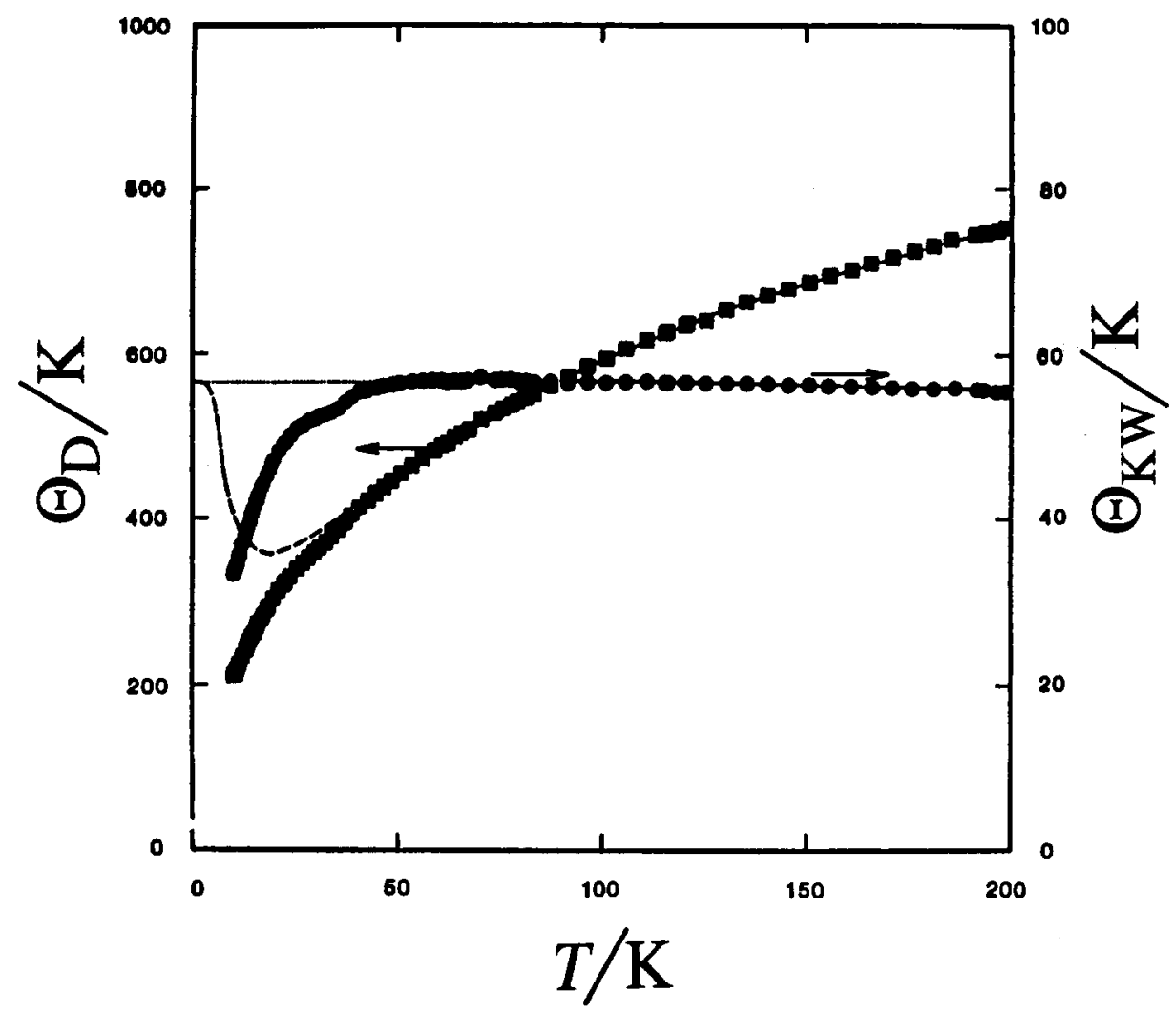

Fig. 3. A comparison of charactcristic temperatures. The Debye $\theta_{\mathrm{D}}$ is represented by squares; the Komada-Westrum $\theta_{\mathrm{KW}}$ by circles. The dashed lines represent the extrapolation of the lattice curve (depicted as $\theta_{\mathrm{D}}$ ) to near $0 \mathrm{~K}$ from the very constant values of $\theta_{\mathrm{KW}}$ at higher temperatures. (Excellent resolution of the transition and shoulder was obtained with $\theta_{\mathrm{KW}}$.) The data are from Ref. [21].

This serves to confirm the essential validity of both methods despite the very different approaches. The Komada-Westrum approach has been used in the resolution of magnetic transition (in grunerite) [22], Schottky (in lanthanides and other compounds), and even of electron delocalization phenomena (in deerite) [22].

Finally, in concluding this review of thermophysically relevant evaluations and calculations we cannot fail to take that Mimkes [23] in a specially invited lecture at this conference entitled "Intermarriage - Phase Diagrams of Science" has extrapolated regular solution models dealing with atomic pair probabilities to test their applicabilities to (human) sociological concerns. The formation of ghettos is equivalent to the miscibility gap in alloys; only by high $T$ ("high tolerance") can the two phases be "dissolved" or eliminated. 


\section{References}

[1] W.M. Latimer, J. Am. Chem. Soc., 43 (1921) 1186; 73 (1951) 1480.

[2] G. Grimvall, Int. J. Thermophys., 4 (1984) 363.

[3] I.L. Khodakovski, personal communication, May 1993.

[4] S. Cantor, Science, 198 (1977) 206.

[5] R.R. Wenner, Thermochemical Calculations, McGraw Hill, 1941.

[6] Y.-S. Way and J.-S. Chen, J. Phys. Chem. Solids, 53 (1992) 19.

[7] K.-W. Zhang, Proc. CODATA Int. Conf., Beijing, 1993, in press.

[8] S. Benson, cited by R.C. Reid, et al., The Properties of Gases and Liquids, Wiley, 1987.

[9] F. Grønvold and E.F. Westrum, Jr., Inorg. Chem., 1 (1962) 36.

[10] S.W. Kieffer, Rev. Geophys. Space Phys., 17 (1979) 1-19, 20-34, 35-59; 18 (1980) 862-886; 20 (1982) 827.

[11] R.D. Chirico and E.F. Westrum, Jr., J. Chem. Thermodyn., 12 (1980) 71; 13 (1981) 519.

[12] B.H. Justice and E.F. Westrum, Jr., J. Phys. Chem., 67 (1963) 339.

[13] J.A. Sommers, J. Chem. Thermodyn., 8 (1976) 1115; 9 (1977) 1.

[14] E.F. Westrum, Jr., R. Burriel, J.B. Gruber, P.E. Palmer, B.J. Beaudry and W.A. Plautz, J. Chem. Phys., 91 (1989) 4383.

[15] R. Shaviv, E.F. Westrum, Jr., J.B. Gruber, B.J. Beaudry and P.E. Palmer, J. Chem. Phys., 96 (1992) 6149.

[16] N. Komada, Dissertation, University of Michigan, Ann Arbor, MI (cf. Dissertation Abstracts).

[17] E.F. Westrum, Jr. and N. Komada, Thermochim. Acta, 109 (1986) 11.

[18] S.W. Barber and B.J. Martin, Phys. Chem. Solids, 9 (1959) 158.

[19] E.F. Westrum, Jr. and S.W. Barber, unpublished results.

[20] E. Schrödinger, Handbuch der Physik, Vol. X, 1926, p. 275.

[21] N. Komada, E.F. Westrum, Jr., B.S. Hemingway and L.M. Anovitz, J. Chem. Thermodyn., in press.

[22] J.B. Gruber, R. Shaviv, E.F. Westrum, Jr., R. Burriel, B.J. Beaudry and P.E. Palmer, J. Chem. Phys., 98 (1993) 1458.

[23] J. Mimkes, M. Lübbers and H.H. Thomas, Thermochim. Acta, 245 (1994) 1. 